Ferrata Storti Foundation

\title{
Blockade of crizotinib-induced BCL2 elevation in ALK-positive anaplastic large cell lymphoma triggers autophagy associated with cell death
}

\author{
Avedis Torossian, ${ }^{1,2,3}$ Nicolas Broin, ${ }^{1,2,3}$ Julie Frentzel, ${ }^{1,2,3}$ Camille Daugrois, ${ }^{1,2,3,4}$ \\ Sarah Gandarillas, ${ }^{5}$ Talal Al Saati, ${ }^{6}$ Laurence Lamant, $, 1,2,3,4,7,8$ \\ Pierre Brousset, ${ }^{1,2,3,4,7,8}$ Sylvie Giuriato, ${ }^{1,2,3,8,9}$ and Estelle Espinos ${ }^{1,2,3,4,8}$
}

Haematologica 2019

Volume 104(7):1428-1439

${ }^{1}$ Inserm, UMR1037 CRCT, F-31000 Toulouse, France; '2Université Toulouse III-Paul Sabatier, UMR1037 CRCT, F-31000 Toulouse, France; ${ }^{3}$ CNRS, ERL5294 UMR1037 CRCT, F-31000, Toulouse, France; ' ${ }^{4}$ Laboratoire d'Excellence Toulouse-Cancer-TOUCAN, F-31024 Toulouse, France; ${ }^{5}$ Inserm/UPS, US006/CREFRE, F-31000 Toulouse, France; ${ }^{6}$ Inserm/UPS, US006/CREFRE, Service d'Histopathologie, F-31000 Toulouse, France; ${ }^{7}$ Département de Pathologie, IUCT, F-31000 Toulouse, France; ${ }^{8}$ European Research Initiative on ALK-related Malignancies (ERIA), Cambridge, UK and ${ }^{9}$ Transautophagy: European network for multidisciplinary research and translation of autophagy knowledge, COST Action CA15138, Brussel, Belgium

\section{ABSTRACT}

\begin{abstract}
A naplastic lymphoma kinase (ALK)-positive anaplastic large cell lymphomas are tumors that carry translocations involving the $A L K$ gene at the 2p23 locus, leading to the expression of ALK tyrosine kinase fusion oncoproteins. Amongst hematologic malignancies, these lymphomas are particular in that they express very low levels of Bcell lymphoma 2 (BCL2), a recognized inhibitor of apoptosis and autophagy, two processes that share complex interconnections. We have previously shown that treatment of ALK-positive anaplastic large cell lymphoma cells with the ALK tyrosine kinase inhibitor crizotinib induces autophagy as a pro-survival response. Here, we observed that crizotinibmediated inactivation of ALK caused an increase in BCL2 levels that restrained the cytotoxic effects of the drug. BCL2 downregulation in combination with crizotinib treatment potentiated loss of cell viability through both an increase in autophagic flux and cell death, including apoptosis. More importantly, our data revealed that the blockade of autophagic flux completely reversed impaired cell viability, which demonstrates that excessive autophagy is associated with cell death. We propose that the downregulation of BCL2 protein, which plays a central role in the autophagic and apoptotic machinery, combined with crizotinib treatment may represent a promising therapeutic alternative to current ALK-positive anaplastic large cell lymphoma treatments.
\end{abstract}

\section{Introduction}

Anaplastic large cell lymphoma (ALCL) is an aggressive subtype of peripheral Tcell non-Hodgkin lymphoma that accounts for $10-15 \%$ of childhood lymphomas. ${ }^{1}$ Two systemic forms of ALCL are currently recognized based on the 2016 revised World Health Organization (WHO) lymphoma classification, ${ }^{2}$ according to the presence or absence of chromosomal translocations involving the anaplastic lymphoma kinase $(A L K)$ gene at the $2 \mathrm{p} 23$ locus. Almost $90 \%$ of ALK-positive ALCL in children carry a characteristic $\mathrm{t}(2 ; 5)(\mathrm{p} 23 ; \mathrm{q} 35)$ chromosomal translocation, leading to the intracellular expression of the oncogenic fusion protein nucleophosmin (NPM)-ALK. ${ }^{3}$ ALK fusion proteins are constitutively active tyrosine kinases that lead to the activation of several downstream pathways, such as MEK/ERK, STATs and PISK/AKT/mTOR, which result in abnormal proliferation and cell survival. ${ }^{4,5}$

Current standard therapies for ALCL in children and adolescents, most common- 
ly based on short-pulse chemotherapy courses, reach event-free survival rates of $70 \% .{ }^{1}$ However, some patients still fail therapy and continued therapeutic improvements with reduced toxicity are being pursued. Recently, targeted therapy against ALK using the dual ALK/MET tyrosine kinase inhibitor crizotinib has been shown to be effective in relapsed/resistant ALK-positive ALCL., ${ }^{6,7}$ However, as reported for other tyrosine kinase inhibitors, escape mechanisms which allow cancer cells to overcome the effects of crizotinib have already been described in ALK-positive non-small cell lung carcinoma (NSCLC), inflammatory myofibroblastic tumors (IMT), and ALCL patients.,

Recently, several studies performed in ALK-associated cancers, including ours in ALCL, demonstrated that macroautophagy (hereafter referred to as "autophagy") is induced following treatments with ALK tyrosine kinase inhibitors and acts as a cell survival-promoting mechanism restraining the cytotoxic effects of the drugs. ${ }^{10}$ Autophagy is a highly-conserved catabolic pathway and a dynamic process (autophagic flux) that is responsible for double membrane autophagosome synthesis, delivery of autophagic substrates to the lysosomes, and degradation of autophagic substrates inside lysosomes. ${ }^{11}$ In cancer, autophagy is often described as a cell survival-promoting mechanism, but cell death-promoting roles have also been reported according to the stage of cancer development and treatments administered. ${ }^{12-14}$ Thus, autophagy is considered to be a multifaceted regulator of cell death and outstanding questions remain as to how it interacts with other forms of cell death, including apoptosis and necrosis/necroptosis. ${ }^{15}$

Indeed, the inter-relationship between these different forms of cell death is extremely complex and the common underlying molecular machinery makes it difficult to distinguish one form from another. ${ }^{15-17}$ BCL2-family proteins regulate all major types of cell death, including apoptosis, necrosis and autophagy, thus operating as nodal points at the convergence of multiple oncological pathways. ${ }^{18}$ The pro-survival BCL2 family members, BCL2 and BCL2L1 (BCL-XL/S) have been reported to directly inhibit autophagy by binding to a BH3-like domain of the BECN1 autophagy protein. ${ }^{19}$ Lindqvist et al. have, however, recently proposed that pro-survival BCL2 family members indirectly inhibit components of the autophagy pathway by inhibiting the activation of BAX and BAK. ${ }^{20,21}$

Deregulation of BCL2 and other anti-apoptotic proteins has been demonstrated to be an important resistance mechanism to treatments in solid tumors and hematologic malignancies. ${ }^{18}$ It has been proposed that the oncogenic properties of BCL2 could originate not only from its ability to block apoptosis but also from its capacity to inhibit BECN1-dependent autophagy, thus preventing BECN1-dependent autophagic cell death. ${ }^{22}$ This further supports BCL2 as a critical target for cancer treatment, and numerous BCL2 targeting strategies are being developed for therapeutic applications. These include pharmacological inhibitors, such as the highly selective BCL2 inhibitor venetoclax (ABT-199)23,24 and, more recently, BCL2-targeted DNAi ${ }^{25}$ or microRNA mimics like miR-34a. ${ }^{26-28}$

Here, we show for the first time that ALK inactivation in ALK-positive ALCL induces an increase in BCL2 levels, which could contribute to therapeutic failures of current ALK-targeted therapies. We found that BCL2 downregulation strongly potentiates the cytotoxic effects of crizotinib both in vitro and in vivo, by increasing the intensity of the autophagic flux as a support for subsequent cell death. Our data strongly suggest that the BCL2 protein, acting at the crossroads between different forms of cell death, is the keystone of an escape mechanism in therapeutically-challenged ALK-positive ALCL. Therefore, BCL2 downregulation in combination with crizotinib treatment could significantly improve clinical outcome in ALK-positive ALCL patients.

\section{Methods}

\section{Human cell lines}

KARPAS-299, COST, and SU-DHL-1 ALK-positive ALCL cell lines which express the NPM-ALK fusion protein were originally obtained from DSMZ (German Collection of Microorganisms and Cell Culture, Braunschweig, Germany) or established in our laboratory. ${ }^{29}$ The ALK-negative ALCL cell line FE-PD was a kind gift from Dr. K. Pulford (Oxford University, Oxford, UK). Cells were cultured as previously described..$^{30}$ The mRFP-EGFP-LC3 KARPAS299 cell line (clonal cell population) was established in our laboratory (Online Supplementary Methods). This study was carried out in accordance with protocols approved by the institutional review board, and the procedures followed were in accordance with the Declaration of Helsinki of 1975, as revised in 2000.

\section{Chemicals}

Crizotinib (PF-2341066) was synthesized and purchased from @rtMolecule (Poitiers, France). Chloroquine was purchased from Sigma-Aldrich (St. Louis, MO, USA). Rapamycin was provided with the Cyto-ID Autophagy detection kit (Enzo Life Sciences, Switzerland). Stock solutions of crizotinib $(500 \mu \mathrm{M})$, chloroquine $(50 \mathrm{mM})$ were prepared in phosphate buffered saline (PBS). Stock solutions of rapamycin $(500 \mu \mathrm{M})$ were prepared in dimethyl sulfoxide (DMSO).

\section{Cell viability determination by MTS colorimetric measurements}

The CellTiter 96AQueus One Solution Cell Proliferation assay (Promega, Fitchburg, WI, USA) was used according to the manufacturer's instructions to follow the growth of the cells and determine the number of viable cells.

\section{Cell death measurements using annexin $\mathrm{V} /$ propidium iodide staining}

Analysis of dying cells was carried out using annexin V (Annexin V-PE) and propidium iodide (PI) staining (BD Biosciences), according to standard protocols. This was followed by flow cytometry using a MACSQUANT MQ10 (Miltenyi Biotec, Santa Barbara, CA, USA). Results were analyzed using FlowJo software (v.10, BD Biosciences).

\section{Flow cytometric quantification of autophagic flux}

Autophagic flux was either assessed on a stable clonal population of mRFP-EGFP-LC3-expressing KARPAS-299 cells, recently generated in our laboratory (Online Supplementary Methods) or on whole cell population of KARPAS-299, SU-DHL-1 and COST cells using the Cyto-ID- based procedure, according to the manufacturer's instructions (Enzo Life Sciences, Switzerland).

\section{Confocal microscopy}

Cells were fixed (20 min, 4\% PFA) on polylysine-coated slides $(0.01 \%)$ and stained with DAPI using ProLong ${ }^{\circledR}$ Gold Antifade Mountant with DAPI (Thermofisher, Waltham, MA, USA). Analysis by confocal microscopy (LSM-780, Zeiss) was performed 
in order to observe fluorescent signals in mRFP-EGFP-LC3 KARPAS-299 cells. Image analysis was performed using the ImageJ software (US National Institutes of Health, Bethesda, MD, USA).

\section{Statistical analysis}

Results are presented as mean values \pm Standard Error of Mean (SEM) from at least three independent experiments, unless otherwise indicated. Determination of statistical significance was performed using the two-tailed Student $t$-test for side-by-side comparison of two conditions. Statistical analyses of xenografted tumor growths were performed using the two-way analysis of variance (ANOVA) followed by the Bonferroni test using GraphPad Prism 6 software (GraphPad, San Diego, CA, USA). For all tests, statistical significance is indicated by: ${ }^{*} P \leq 0.05$; ${ }^{*} P \leq 0.01$; ${ }^{* * *} P \leq 0.001 ;{ }^{* * *} P \leq 0.0001$.

\section{Results}

\section{BCL2 levels inversely correlate with ALK expression and ALK activity in anaplastic large cell lymphoma cells}

Whereas overexpression of BCL2 is a classical feature in cancers, including hematopoietic tumors, previous studies reported very low levels of BCL2 in ALK-positive primary tissue samples. ${ }^{31,32}$ To investigate whether there was a link between ALK and BCL2 expression, we first checked the relative abundance of these two proteins in three ALKpositive and one ALK-negative cell lines (Figure 1A). BCL2 protein levels were found to be very low in the three ALKpositive cell lines tested (KARPAS-299, SU-DHL-1, and COST) but were much more abundant in the ALK-negative (FE-PD) cell line, suggesting an inverse correlation between the expression of NPM-ALK and BCL2. We then monitored BCL2 levels following treatment with the crizotinib compound for 24 hours (h) at the known plasmatic dose (500 nM) observed in patient ${ }^{30}$ (Figure 1B). Our data indicate that the abolition of ALK kinase activity resulted in a consistent and highly reproducible increase in BCL2 levels in ALK-positive cells, with no effect in ALK-negative cells (for quantification, see Online Supplementary Figure S1A). ALK knockdown, through the use of a targeted siRNA, confirmed these results, showing an ALK-dependent increase in BCL2 levels (Figure 1C and Online Supplementary Figure S1B for quantification). Finally, as crizotinib is known to inhibit both ALK and MET tyrosine kinases, ${ }^{6}$ we then checked the effects of the specific molecular downregulation of MET, using a targeted siRNA, on BCL2 cellular levels. We did not observe any increase in BCL2 levels following MET knockdown (Online Supplementary Figure S2). Thus, this result rules out the contribution of MET inactivation in the increase of BCL2 levels following crizotinib treatment. Altogether, these data support the existence of a strictly ALK-dependent BCL2 repression mechanism at work. Therefore, treating ALK-positive ALCL with crizotinib impairs this mechanism and leads to the re-expression of the BCL2 oncogene.

\section{Increased BCL2 levels limit the cytotoxic effects of crizotinib}

We next asked whether crizotinib-mediated increase in BCL2 levels could limit the cytotoxic effects of the drug. We thus performed viability assays, cell cycle analyses, and Annexin V/PI staining in cells that were knocked down or not for BCL2, and treated or not with crizotinib (Figure 2). BCL2 knockdown (confirmed by western blot analysis) (Online Supplementary Figure S3) was achieved by RNA interference using either a targeted siRNA directed against BCL2 mRNA, or miR-34a mimics, another promising BCL2 targeting strategy for clinical application. ${ }^{26-28}$ Viability assays showed that BCL2 knockdown, which restrained BCL2 elevation in crizotinib-treated cells (without up-regulating other BCL2 family isoforms, including MCL1, BCL-XL/S and BCL-W; data not shown), significantly enhanced the effects of crizotinib, as only $20 \%$ of siBCL2-transfected cells remained viable after $72 \mathrm{~h}$ when compared to approximately $50 \%$ of siCTL- transfected cells (Figure 2A). More pronounced effects on cell viability were obtained when BCL2 knockdown was achieved using miR-34a mimics, as only $6 \%$ of cells were viable at the end of the combined treatment.

We then explored whether this loss in cell viability involved a decrease in cellular growth, by analyzing the effects of BCL2 silencing and/or crizotinib treatment on cell cycle distribution (Figure 2B). We found that BCL2 downregulation using a targeted siRNA did not increase either the potent G1-S phase cell cycle arrest or the number of cells in sub-G1 phase observed following crizotinib treatment. On the contrary, the use of miR-34a mimics induced per se a blockade in G1 phase and an increase in the number of cells in sub-G1 phase, which were further potentiated upon crizotinib addition (Figure $2 B$ ).

To better assess the effects of BCL2 knockdown on cell death, we performed Annexin V/PI staining. Our data first showed that crizotinib treatment $(500 \mathrm{nM}, 72 \mathrm{~h})$ induced apoptosis, as reflected by a significant increase in the number of annexin V-stained cells in siCTL and miR-Neg conditions (Figure 2C). Additionally, and in agreement with the sharp loss in cell viability observed in response to combined treatments, we observed that BCL2 knockdown triggered an increase in apoptotic cell death in crizotinib-treated cells, as revealed by both a significant increase in the number of annexin V-stained cells and an activation of caspase 3/7 (Online Supplementary Figure S4).

Taken together, our data indicate that the cytotoxic effects of crizotinib in ALK-expressing ALCL cells are restricted through an elevation of BCL2 levels. Indeed, we demonstrate that BCL2 downregulation in addition to crizotinib treatment potentiates crizotinib-induced loss in cell viability essentially through an increase in apoptotic cell death. Of note, similar results on cell viability and apoptosis were obtained using SU-DHL-1, another ALKpositive ALCL cell line (Online Supplementary Figure S5).

\section{BCL2 downregulation enhances crizotinib-triggered autophagic flux}

Besides its role in apoptosis, BCL2 is also a known inhibitor of autophagy, ${ }^{19-22}$ a process that has been described to influence cell death mechanisms in many cancers following drug treatment. ${ }^{12,15,33}$ Since we recently showed that ALK inactivation induced autophagy endowed with pro-survival properties in ALK-positive ALCL cell lines, ${ }^{30}$ we investigated whether concomitant elevation of BCL2 could account for the cytoprotective function of the autophagic process in these conditions. To address this question, we generated a modified and clonal KARPAS-299 cell line stably transfected with a transgene encoding the LC3 protein (microtubule-associated protein 
1 light chain 3), an early marker of autophagosomes, coupled to both RFP and EGFP (Online Supplementary Methods and Online Supplementary Figure S6). The expression of this tandem fluorescently-tagged LC3 reporter protein enabled flow cytometric quantification and confocal microscopybased analysis of autophagic flux. In accordance with our previous study, ${ }^{30}$ we observed that crizotinib induced autophagic flux in a dose-dependent manner, as revealed by an increase in the percentage of cells that exhibited high RFP/EGFP fluorescence ratios (Figure 3A). More importantly, and in keeping with the known function of BCL2 in autophagy downregulation, ${ }^{19-22}$ we found that BCL2 knockdown, using either siBCL2 (Figure 3A and B) or miR-34a mimics (Figure $3 \mathrm{~A}$ and $\mathrm{C}$ ), strongly increased the percentage of cells with high RFP/EGFP ratios, indicative of a higher autophagic flux, which was observed both in basal and crizotinib-treatment conditions. We confirmed these results by performing two other assays to monitor and quantify autophagic flux in the KARPAS-299 whole cell population (Online Supplementary Figures S7S9), and in two other ALK-positive ALCL cell lines (Online Supplementary Figure S10). Altogether our results strongly indicate that BCL2 plays a key role in restraining both the basal- and crizotinib-induced autophagic flux in ALK-positive ALCL.

\section{Enhanced autophagic flux induced by BCL2 downregulation and crizotinib treatment is associated with impaired cell viability}

To elucidate whether this substantial increase in autophagic flux upon combined crizotinib treatment and BCL2 downregulation could be involved in the increased loss of cell viability observed under the same conditions, we followed the survival of cells in which the autophagic machinery was impaired through the knockdown of ULK1, a key factor involved in the very early stages of the autophagy process. ${ }^{34}$ mRFP-EGFP-LC3 KARPAS-299 cells were transfected with siRNA directed against ULK1

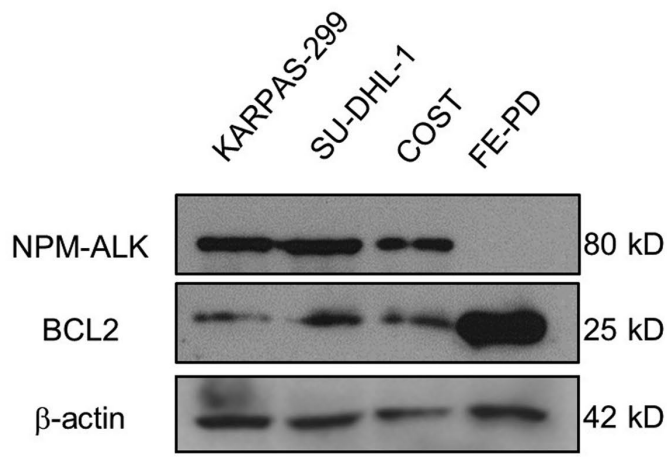

B
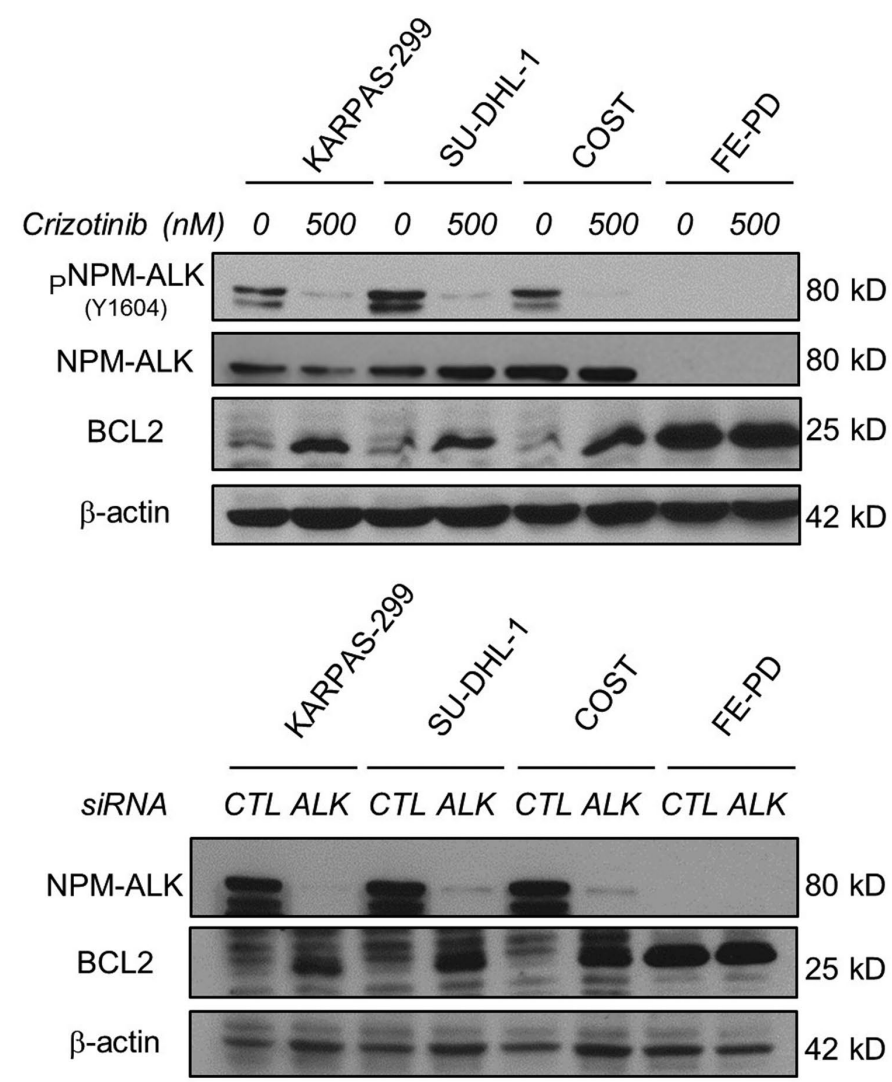

Figure 1. BCL2 levels inversely correlate with NPM-ALK expression and ALK tyrosine kinase activity in anaplastic large cell lymphoma (ALCL) cells. (A) Western blot showing NPM-ALK and BCL2 protein levels in ALK-positive (KARPAS-299, SU-DHL-1, COST) and ALK-negative (FE-PD) ALCL cell lines. $\beta$-actin served as the internal control to ensure equal loading. (B) Western blot showing BCL2 protein levels in ALK-positive and ALK-negative ALCL cells following 24 hours ( $h$ ) of treatment with crizo tinib (500 nM). The loss of NPM-ALK tyrosine phosphorylation (P-NPM-ALK, Y1604) served as an internal control to ensure efficiency of crizotinib. (C) Western blot showing NPM-ALK and BCL2 protein levels in ALK-positive and ALK-negative ALCL cells that were transfected with either a negative control siRNA ( $\mathrm{SiCTL}$ ) or a siRNA targeting ALK mRNA (siALK) for $72 \mathrm{~h}$. 
mRNA (siULK1) and/or against BCL2 mRNA (siBCL2), in the presence or absence of crizotinib. ULK1 knockdown (validated by western blot analyses, Online Supplementary Figure S11) efficiently blocked the autophagy process, as revealed by a decrease in both yellow (reflecting autophagosomes) and red (reflecting autolysosomes) punctate staining (Figure 4A). Flow cytometry monitoring of autophagic flux confirmed firstly that the blockade of BCL2 expression increased the number of cells exhibiting high autophagy in both untreated and crizotinib-treated cells (Figure 4B and C), and secondly that ULK1 downregulation successfully blocked the autophagic machinery as it reduced basal autophagy levels and restrained the crizotinib-triggered autophagic flux observed in BCL2-knocked down cells (2-fold decrease). Most interestingly, MTS viability assays showed that the effects of BCL2 downregulation in further reducing cell viability in crizotinib-treated cells was completely reversed by the knockdown of ULK1 (Figure 4D). These results strongly suggest that BCL2 downregulation following crizotinib treatment reinforced autophagic flux, which was deleterious for ALK-positive ALCL cells, and thereby led to a greater loss in viability under these conditions.

To address the question of whether potentiation of the autophagic flux in our model was sufficient to trigger cell death, we next performed experiments with a combination of crizotinib and rapamycin, a well-known mTOR inhibitor and strong inducer of autophagy, namely in ALK-positive tumor cells. ${ }^{35,36}$ We first confirmed that rapamycin alone $(100 \mathrm{nM})$ did induce autophagy in KARPAS-299 cells as $60 \%$ and $75 \%$ of cells harbored a high autophagic flux following $24 \mathrm{~h}$ and $48 \mathrm{~h}$ of treatment, respectively (Figure $5 \mathrm{~A}$ ). We then observed that combining crizotinib and rapamycin treatments resulted in a clear potentiation of the autophagic flux, with more pronounced effects obtained with the lowest dose of crizotinib used in our assays (125 nM). We also found that this potentiation of the autophagic flux was associated with a decrease in cell viability, as measured by MTS colorimetric assays (Figure 5B), an effect that was more pronounced after $48 \mathrm{~h}$ and $72 \mathrm{~h}$ of treatment. However, Annexin-V/PI staining revealed no significant differences in the number of cells undergoing apoptotic cell death when comparing crizotinib treatment alone with crizotinib and rapamycin combined treatment, independently of the dose of crizotinib used and the duration of the treatment (Figure 5C). Altogether these results indicate that excessive autophagy upon crizotinib and rapamycin cotreatment contributes to cell death independently of apoptosis.
A

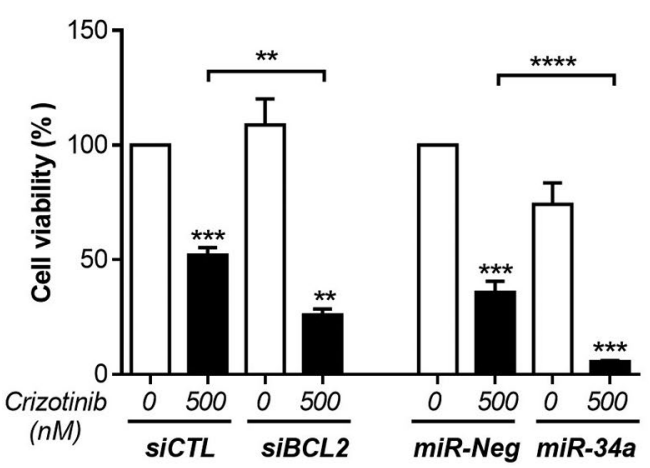

B

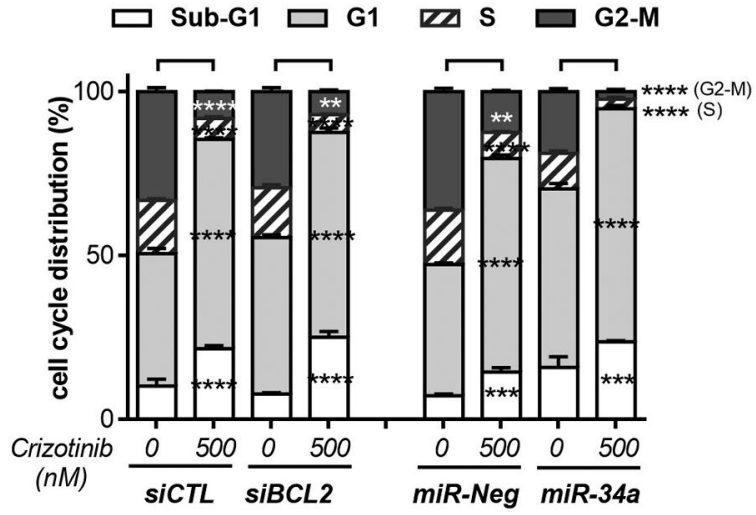

C

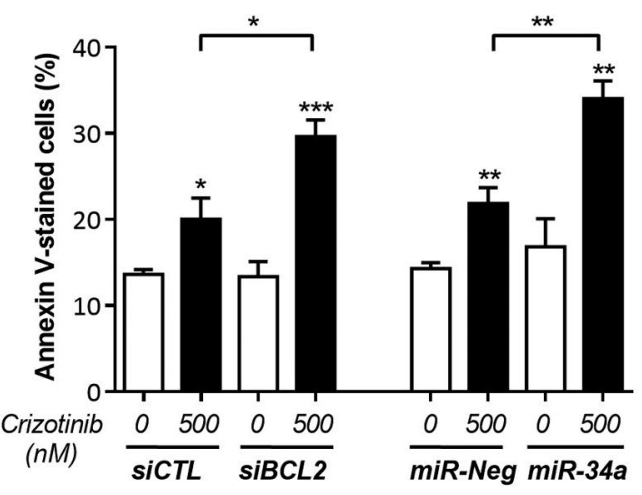

Figure 2. BCL2 downregulation potentiates crizotinib-induced loss in cell viability, which involves an increase in cell death. Twenty-four hours $(h)$ after the transfection of BCL2-targeted interfering RNAs (siBCL-2 or miR-34a mimics), or their corresponding negative controls (siCTL or miR-Neg), KARPAS-299 were treated (or not) with crizotinib (500 nM) for $72 \mathrm{~h}$. (A) Cell viability was assessed by MTS colorimetric measurement. Each set of data was normalized to its related untreated negative control condition (SiCTL or miR-Neg) and represents mean \pm Standard Error of Mean (SEM); $\mathrm{n}=3$. $* * P \leq 0.01 ; * * * P \leq 0.001 ; * * * * P \leq 0.0001$; unpaired Student $t$-test. (B) Flow cytometry analysis of cell cycle. Graph represents the mean percentage of cells in sub-G1, G1, S and G2/M phases. Data represent mean \pm SEM; $n=3$; Statistical analysis was performed by two-way ANOVA with Bonferroni correction; $* * P \leq 0.01$; $* * * P \leq 0.001 ; * * * * P \leq 0.0001$. (C) Flow cytometry analysis of annexin V-positive KARPAS-299 cells. Graph represents the percentage of annexin $\mathrm{V}$-positive cells from six independent experiments \pm SEM. ${ }^{*} P \leq 0.05$; $* * P \leq 0.01 ; * * * P \leq 0.001$; unpaired Student $t$-test. 
MiR-34a-mediated BCL2 downregulation potentiates the antitumoral effects of crizotinib in NOD/SCID mice xenografted with ALK-positive anaplastic large cell lymphoma cells

Finally, we explored whether the combination of BCL2 downregulation and crizotinib treatment would have a significant therapeutic benefit in vivo. To address this question, NOD/SCID mice were xenografted with KARPAS299 cells that were either transfected with miR-Neg or miR-34a mimics. Crizotinib was administered orally for 22 days, during which time tumor growth was monitored (Figure 6A). On the day of sacrifice, tumors were weighed (Figure 6B) and samples were retrieved for immunohistochemistry (IHC) analysis (Figure 6C).

In accordance with the data collected in vitro, we observed that mice xenografted with miR-34a-transfected cells developed significantly smaller tumors than those xenografted with miR-Neg-transfected cells in the presence of crizotinib (Figure 6A and B). As seen in our in vitro viability assays, miR-34a-mediated BCL2 knockdown alone impaired tumor growth, albeit to a lesser extent than with the miR-34a/crizotinib combination. Hematoxylin \& Eosin (HE) staining performed on samples excised from tumors treated with the miR-34a/crizotinib combination also exhibited hallmarks of higher cell fragility (Figure 6C). To confirm our in vitro findings showing higher levels and deleterious effects of autophagy in KARPAS-299 cells under miR-34a/crizotinib combination, we looked at in vivo autophagy activity by performing LC3B and p62 IHC analyses in tissues from the tumor xenografts (Figure 6C and Online Supplementary Figure S12), as previously reported. ${ }^{37,38} \mathrm{We}$ observed that dot-like patterns of both LC3B and p62 staining increased strongly and significantly in cells that received the combined miR-34a/crizotinib treatment in comparison with the single treatment. These results are consistent with increased autophagy activity.

\section{Discussion}

Our study is the first to report that the expression of ALK and BCL2, two major oncogenes, are inversely correlated in ALK-positive ALCL through an ALK-dependent BCL2 repression mechanism. Indeed, we demonstrated that BCL2 levels increased following either the pharmacological inhibition (crizotinib treatment) or the siRNA-targeted knockdown of ALK (siALK transfection) in ALK-

A

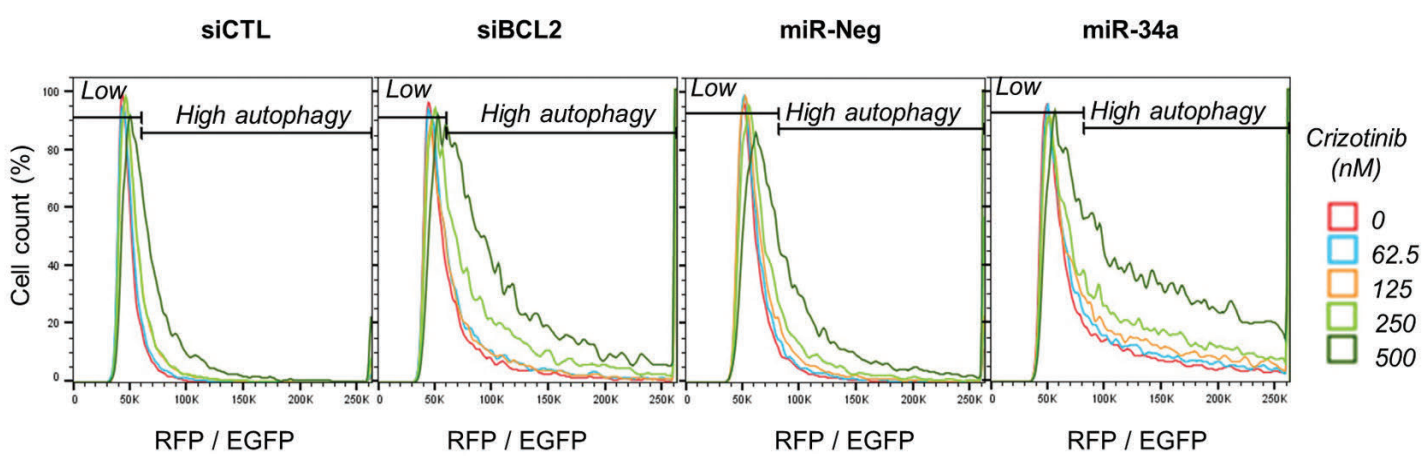

B

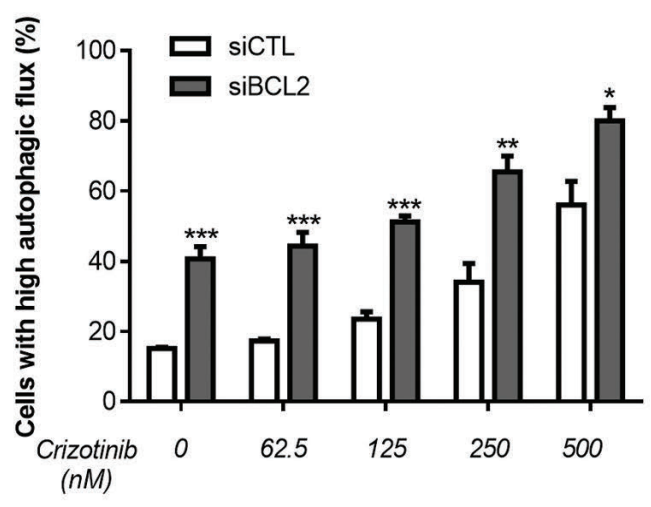

C

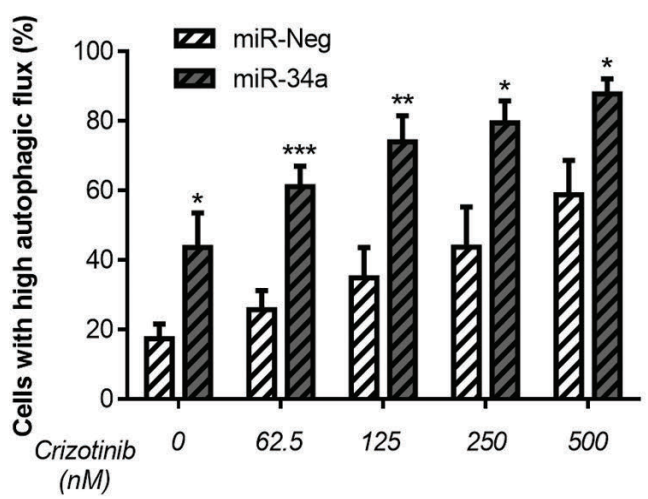

Figure 3. BCL2 downregulation enhances crizotinib-triggered autophagic flux. (A) Flow cytometry analysis of autophagic flux following the knockdown of BCL2 and crizotinib treatment in KARPAS-299 cells expressing a tandem fluorescently-tagged LC3 reporter protein. mRFP-EGFP-LC3 KARPAS-299 cells (described in Online Supplementary Methods) were transfected with either negative controls (siCTL or miR-Neg) or with siBCL2 or miR-34a mimics. Twenty-four hours (h) later, transfected cells were treated or not with increasing doses of crizotinib (0 to $500 \mathrm{nM})$ for a further $48 \mathrm{~h}$. Induction of autophagic flux was analyzed by monitoring the RFP/EGFP fluorescence ratio in individual cells. Cells were split into two groups based on their relative RFP/ EGFP fluorescence ratios: cells with low/basal autophagic flux and cells with high/induced autophagic flux. A representative experiment is shown. (B and C) Histograms representing the percentage of cells with a RFP/EGFP ratio reflective of high autophagic flux, from $n=5$ (siBCL2) or $n=3$ (miR-34a mimics) independent experiments \pm Standard Error of Mean; $* P \leq 0.05$; $\star * P \leq 0.01$; $* * * P \leq 0.001$; unpaired Student's $t$-test. 
positive ALCL cells. These data are consistent with and complete previous studies reporting a lack of BCL2 protein expression in primary tissue samples of ALK-positive ALCL $^{31,32}$ and low mRNA levels in the ALK-positive ALCL cell line, KARPAS-299. ${ }^{39}$ This observation in a cancerous environment seems paradoxical but is balanced by the fact that these tumor cells over-express MCL1, through a molecular mechanism that involves miR-29a. ${ }^{32,39,40}$ In the present study, to rule out a potential compensation of BCL2 downregulation by BCL2 isoforms in crizotinibtreated cells, we checked the expression of MCL1, BCL-XL/S and BCL-W and did not find any significant
A
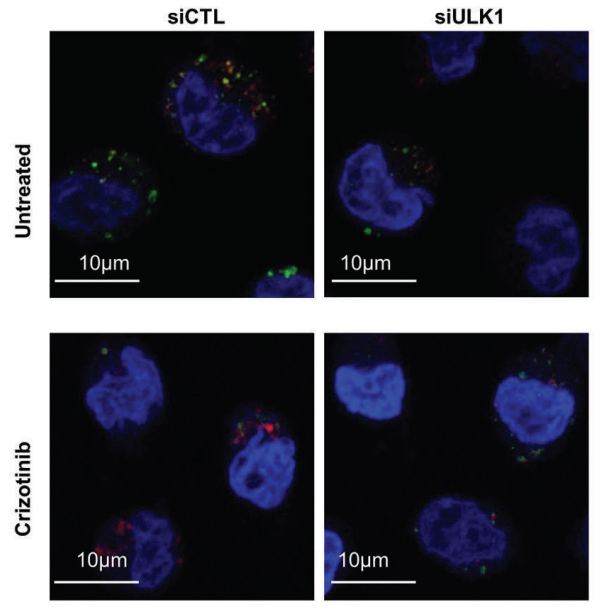

siBCL2
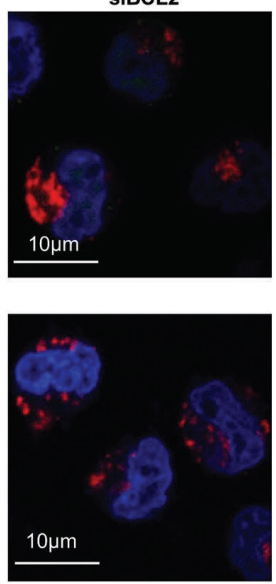

siBCL2 + siULK1
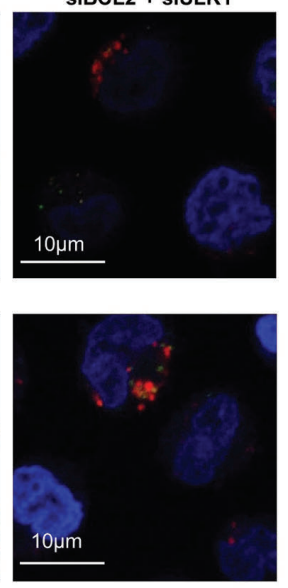

B

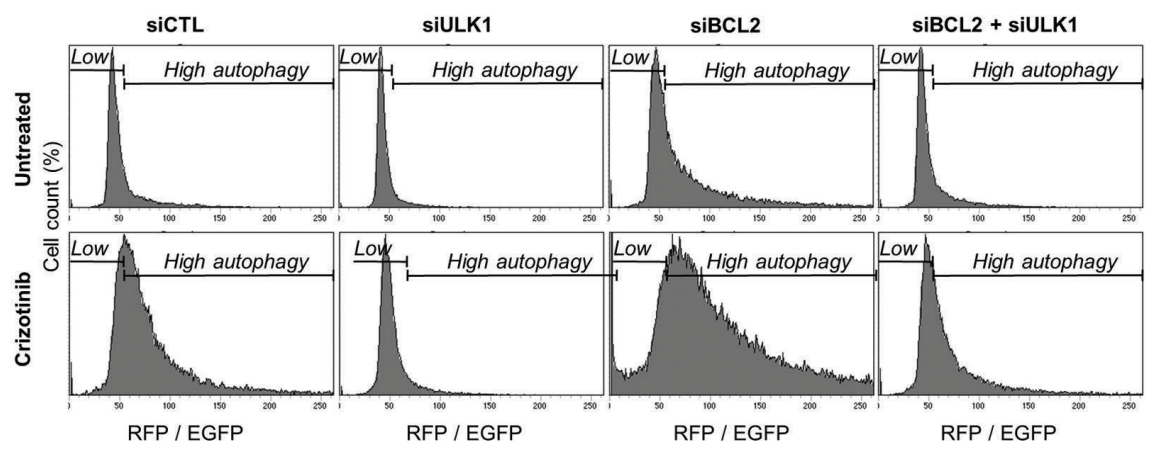

C
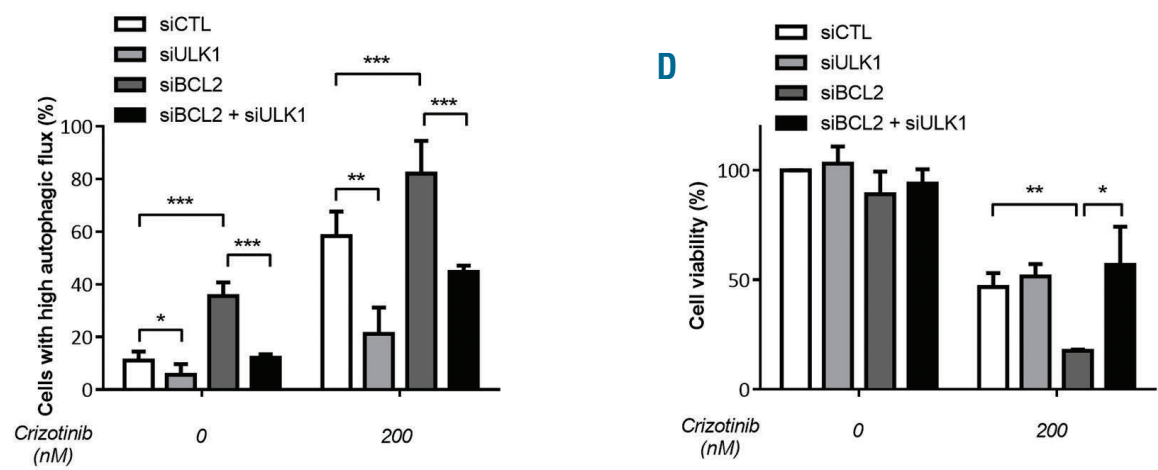

Figure 4. Enhanced autophagic flux induced by BCL2 downregulation and crizotinib treatment is associated with impaired cell viability. Twenty-four hours (h) after ULK1 knockdown either alone or in combination with BCL2 knockdown, mRFP-EGFP-LC3 KARPAS-299 cells were treated or not with crizotinib (200nM) for a further $72 \mathrm{~h}$. Representative data of (A) confocal microscopy and (B) flow cytometry analysis of autophagic flux are shown. (C) Histograms representing the percentage of cells with high autophagic flux from five independent experiments \pm Standard Error of Mean (SEM); $* P \leq 0.05 ; * * P \leq 0.01 ; * * * P \leq 0.001$; unpaired Student $t$-test. (D) Cell viability was assessed by MTS colorimetric measurements in the same experimental conditions. Data represent mean $\pm S E M$; $\mathrm{n}=3$; $* P \leq 0.05$; $\star \star P \leq 0.01$; unpaired Student $t$-test. 
variations in the levels of all of these isoforms when comparing crizotinib-treated siCTL- to siBCL2-transfected cells (data not shown). We then demonstrated that increased BCL2 levels limit the cytotoxic effects of crizotinib. To do so, we used two molecular approaches to target BCL2: i) a specific BCL2 siRNA; and ii) miR-34a mimics, which have been reported to reduce cell viability and induce apoptosis through efficient BCL2 downregulation. ${ }^{41,42}$ We found that miR-34a mimics potentiated crizotinib cytotoxicity more efficiently than siBCL2, an effect that could be attributed to the versatile role of miR-34a in regulating the expression of myriads of targets that include not only proteins involved in apoptosis, such as BCL2, but also proteins controlling cell cycle and other processes that are necessary for cell viability. ${ }^{26,28}$ The broad anti-oncogenic activity of miR-34a is nowadays considered to be a clear advantage for the treatment of multigenic diseases such as cancer and justifies the development of miR-34a mimics deliverybased strategies to combat cancer cells. ${ }^{27,43,44}$ MRX34, a
A

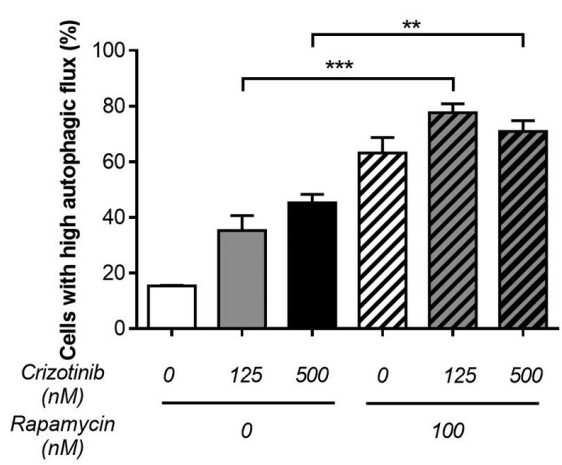

B

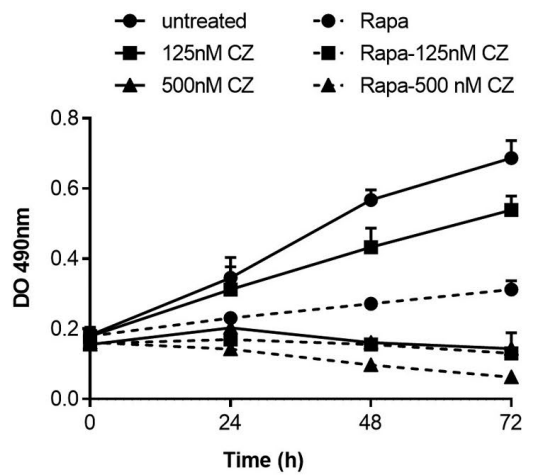

$48 \mathrm{~h}$

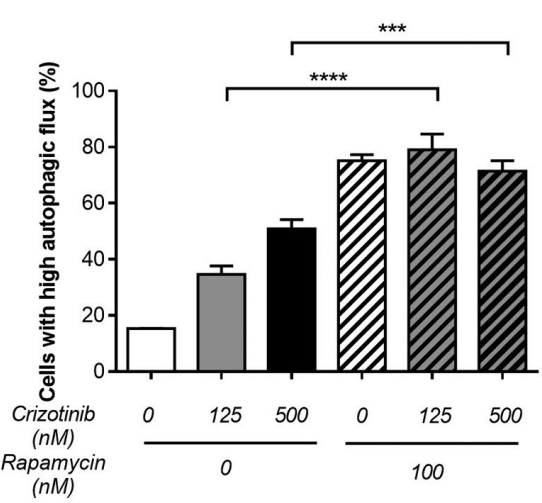

(nM) 
liposome-based miR-34a mimic is the first miRNA mimic to be used in clinical studies and has already been evaluated in phase I clinical trials in patients with advanced solid tumors. ${ }^{45}$ The combination of miR-34a mimics with other anti-cancer agents could thus represent a novel way to counteract therapeutic failure. In addition, we carried out experiments with the BH3-mimetic venetoclax. We treated KARPAS-299 ALCL cells with venetoclax concentrations (from $10 \mathrm{nM}$ to $25 \mu \mathrm{M}$ ), which were described by Souers et al. for cells with high and low
A

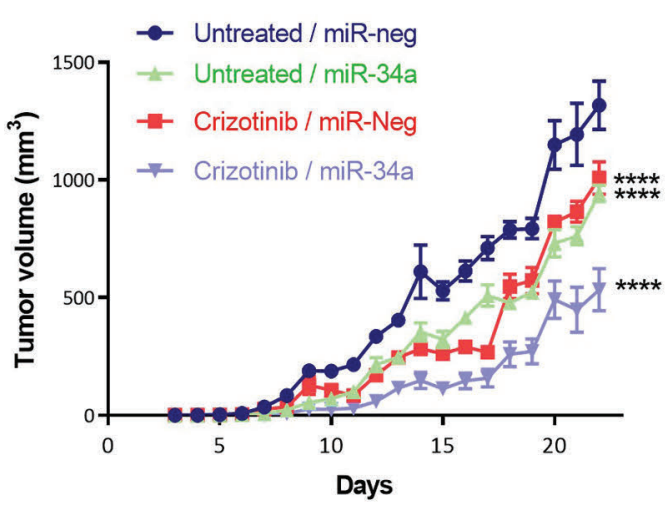

C
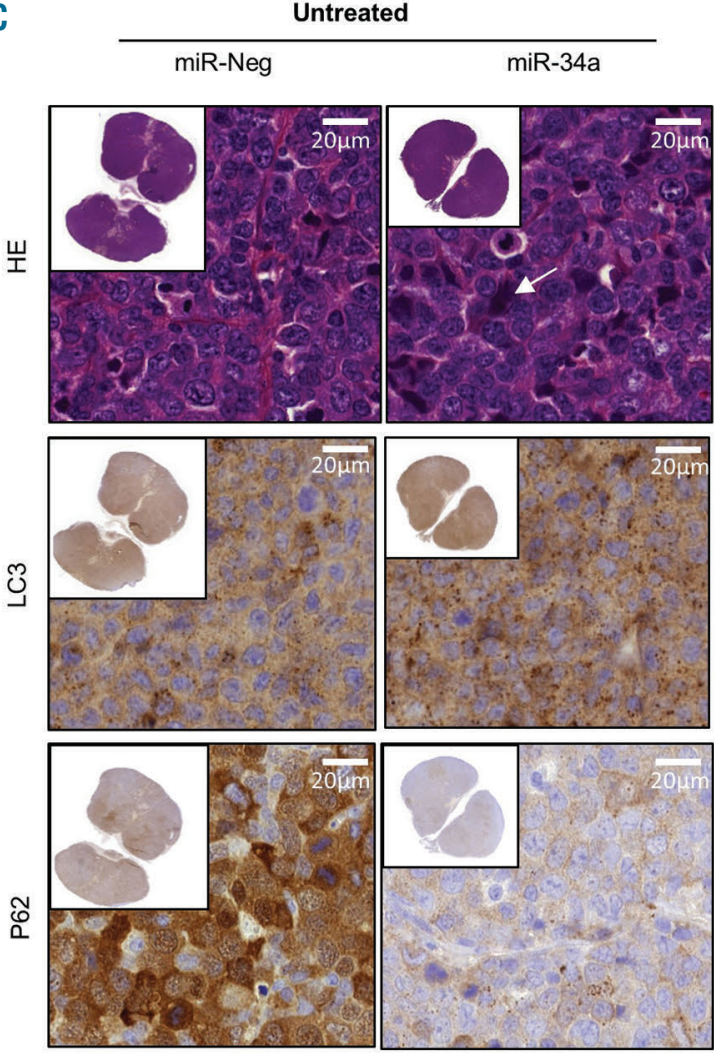

B
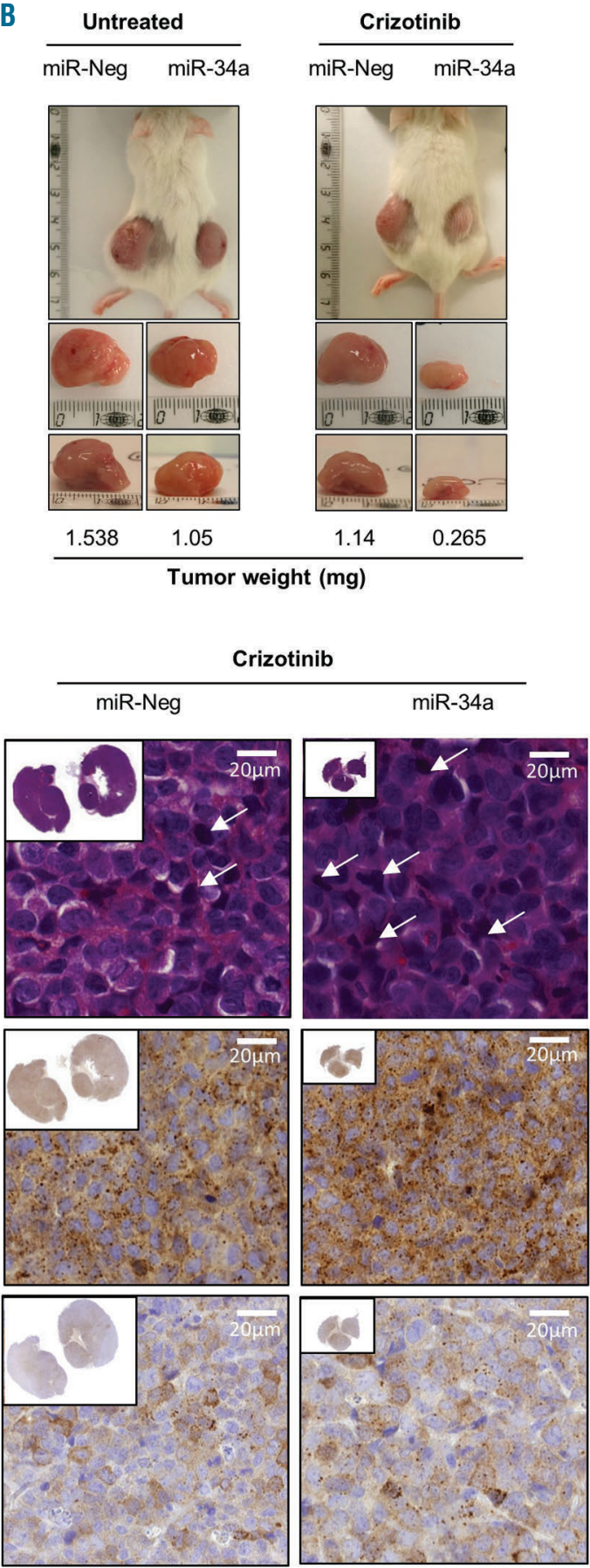

Figure 6. miR-34-mediated BCL2 downregulation potentiates the antitumoral effects of crizotinib in vivo. (A) ALK-positive KARPAS-299 cells, transfected with either a negative control microRNA (miR-Neg) or miR-34a mimics (miR-34a), were injected subcutaneously into the left or right flank of 16 NOD/SCID mice, respectively. Eight of these mice received oral crizotinib $(2.5 \mathrm{mg} / \mathrm{kg})$ for 22 days, while the remaining eight received vehicle. Tumor volume was evaluated over time by caliper measurements and was reported as means \pm Standard Error of Mean (SEM). Statistical analysis was performed by two-way ANOVA with Bonferroni correction; $* * * * P \leq 0.0001$. (B) Representative tumors resected from mice xenografted with miR-Neg or miR-34a cells that received either vehicle or crizotinib treatment (scale in $\mathrm{cm}$ ). Their weights are indicated (mg). (C) Micrographs showing Hematoxylin \& Eosin (HE), anti-LC3B and anti-P62 stainings of excised miR-Neg or miR-34a tumors that in addition received either vehicle or crizotinib treatment (scale bars: $20 \mu \mathrm{m}$, inset HE staining $5000 \mu \mathrm{m}$ ). Arrows indicate cells with phenotypic hallmarks of nuclear piknosis and general cellular fragility. 
levels of BCL2, respectively. ${ }^{23}$ We found low concentrations of venetoclax to have no impact either on cell viability or autophagy. Conversely, high doses were found to be highly toxic, even in the absence of crizotinib, a result most likely attributable to off-target effects (data not shown). Thus, in accordance with literature highlighting the efficiency of this compound in the treatment of tumors harboring high expression of BCL2 but its uncommon use in lymphoma, we found that the pharmacological inhibition of BCL2 with venetoclax in KARPAS-299 ALK positive cells did not recapitulate the effects of BCL2 targeted downregulation, a result that should stimulate further investigation.

Our team previously demonstrated that treatment of ALK-positive ALCL cells with crizotinib induces an autophagic flux endowed with pro-survival properties. ${ }^{30}$ In the present study, we further demonstrated that crizotinib-induced autophagy was associated with an upregulation of BCL2, which limits the cytotoxic effects of the drug. Indeed, BCL2 knockdown combined to crizotinib treatment led to a profound loss of cell viability that was found to be due to an increase in apoptosis. This did not, however, exclude the occurrence of another type of cell death. Indeed, in addition, our data showed that crizotinib/BCL2 knockdown treatments also led to a significant potentiation of autophagic flux (Figure 7). Several lines of evidence clearly indicate that autophagy is a multifaceted regulator of cell death. ${ }^{12-14}$ However, controversy remains as to whether autophagy directly executes cell death or how it interferes with other forms of death, including apoptosis and necrosis/necroptosis. ${ }^{15}$ In the present study, the contribution of excessive autophagy in the process of cell death was first evidenced by the fact that impairing the autophagic machinery (by the molecular targeting of ULK1) resulted in a complete reversal of the potent loss of cell viability in BCL2-knocked down cells. Secondly, our in vivo data further revealed LC3B and p62 stainings consistent with increased autophagy activity in tumor tissues harvested from ALK-positive ALCL cells xenografted mice submitted to crizotinib and miR34a-mediated BCL2
A

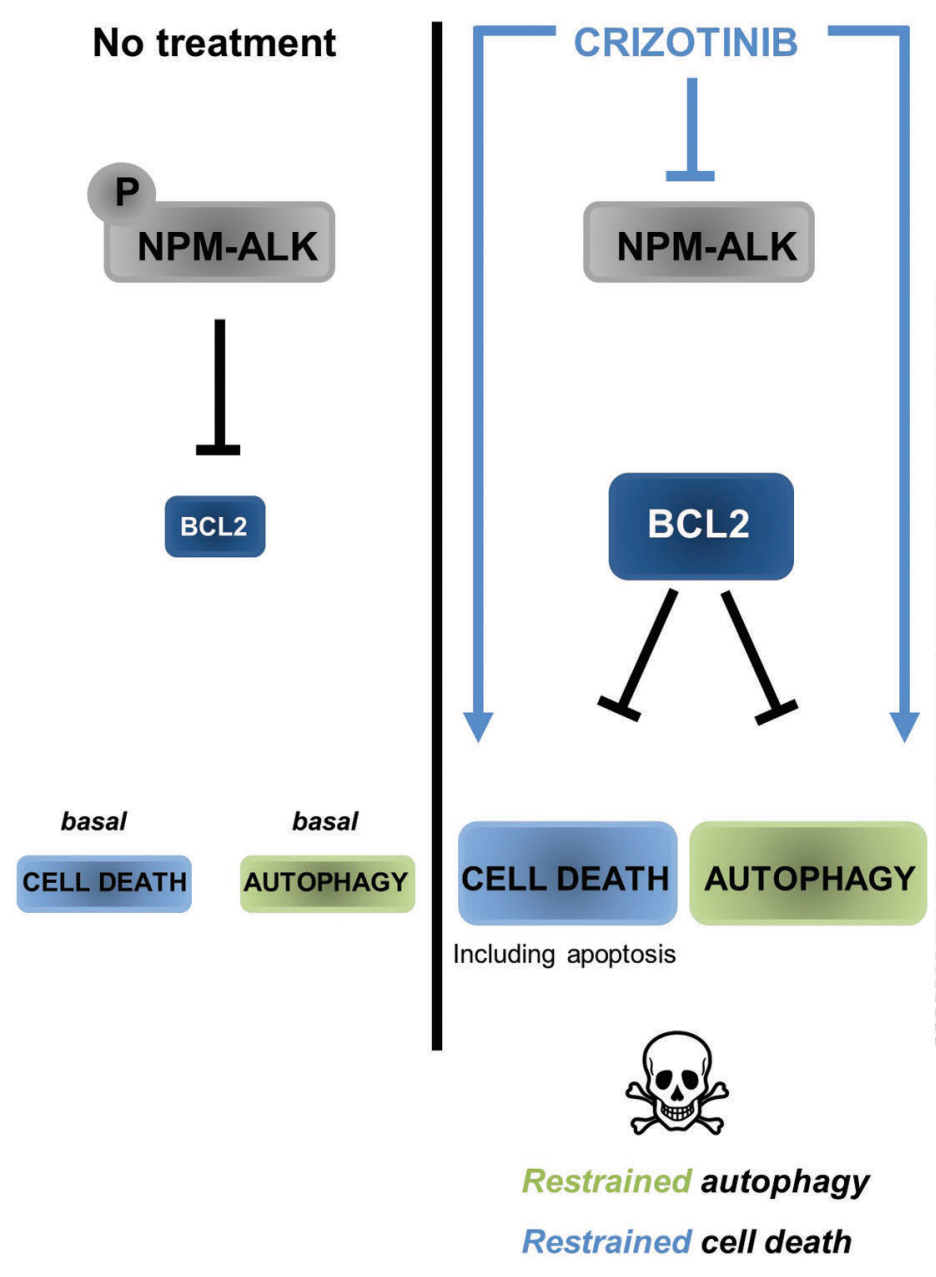

C

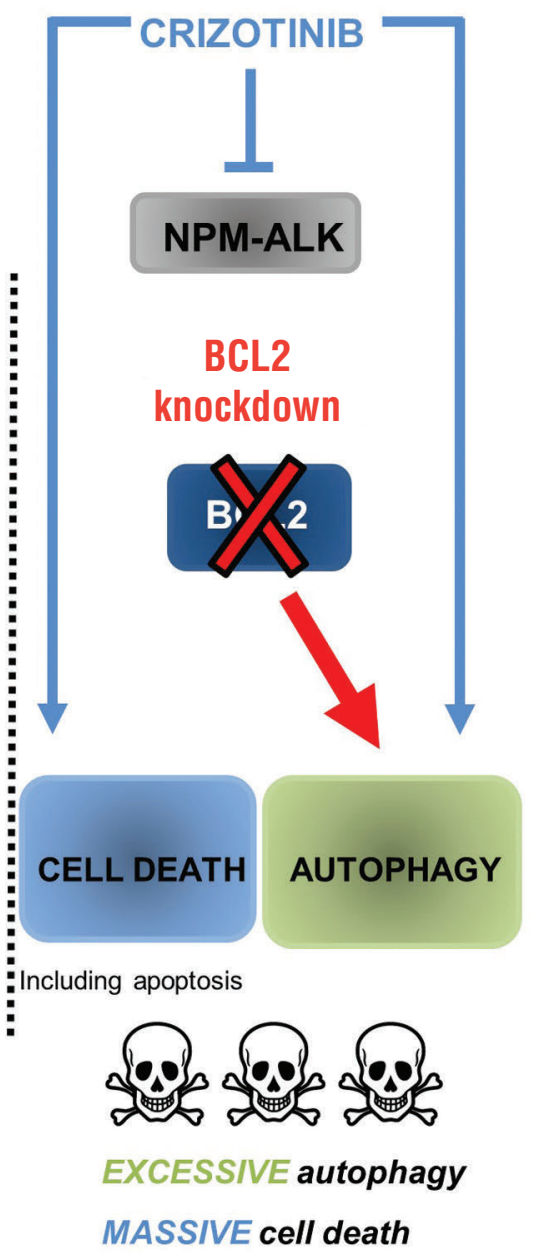

Figure 7. Proposed model of the combined ALK and BCL2 inhibitions on the fate of ALK-positive anaplastic large cell lymphoma (ALCL) cells. (A) An ALK-dependent BCL2 repression mechanism is at work In ALK-positive ALCL cells. (B) Strategies based on the inhibition of ALK activity, such as crizotinib treatment, impair this repression mechanism. This leads to an increase in BCL2 levels that, in turn, limits both cell death and autophagy induction. (C) Blocking crizotinib-induced BCL2 elevation results in a potentiation of the cytotoxic effects of the drug, through both overactivation of autophagic flux and an increase in cell death (including apoptosis and, potentially, other cell death modalities). 
knockdown, which was associated with a remarkable impairment in subcutaneous tumor development. Finally, we used a combination of rapamycin and crizotinib to induce an overactivation of autophagy, which did not rely on BCL2 downregulation. We found that enhanced autophagic flux correlated with impaired cell viability but occurred independently of apoptosis, suggesting the involvement of another cell death modality. Autophagy has, indeed, been shown to provide a scaffold for the necroptotic machinery ${ }^{46}$ and also to determine the means of cell death by serving as a switch between apoptosis and necroptosis. ${ }^{47}$

Further investigations are currently under way to decipher whether excessive autophagy and promotion of cell death upon ALK and BCL2 downregulation in ALK-positive ALCL involve the activation of convergent and interlinked cell death pathways, including autophagy, apoptosis and necroptosis. Either way, our results provide strong evidence for a massive reduction in tumor cell viability following combined ALK and BCL2 inactivation in ALK-positive ALCL, demonstrating that the molecular targeting of BCL2 could widen the therapeutic options for these patients and potentially improve their outcome by reducing the options for cancer cell escape routes.

\section{Acknowledgments}

The authors would like to thank the Inserm and Fondation $A R C$ pour la Recherche sur le Cancer (SGiu and EE), the European Union's Horizon 2020 Research and Innovation Program under the Marie Sklodowska-Curie grant agreement n. 675712 (SGiu and EE), the ANR-16-CE12-018-03 (EE) for grants. The authors also thank the Universite Paul Sabatier (AT), the Ligue Nationale contre le Cancer (JF) and Labex TOUCAN/Laboratoire d'excellence Toulouse Cancer (CD) for individual fellowships. The authors thank the Anexplo-Génotoul platform, Inserm/UPS, US006/CREFRE, Toulouse, France (F. Capilla and C. Salon at the histology facility) and the flow cytometry facility of CRCT/UMR1037/Inserm/UPS/ERL5294 CNRS, Toulouse, France (M. Farcé) for their technical assistance. They thank Dr. G. Mitou (CRCT/ Inserm/UMR1037) for her help in the development of the mRFP-EGFP-LC3 KARPAS-299 cells, Dr S. Kermorgant (Barts Cancer Institute, London, UK) for generously providing tools and advice regarding the c-MET protein and Dr. C. Philippe (CRCT/Inserm/UMR1037/UPS) for her help with cell cycle flow cytometry analyses. They also thank Dr. R. Chiarle, Dr. S. Ducamp (Boston Children Hospital, Boston, USA), Dr. F. Meggetto and Dr. C. Joffre (CRCT/Inserm/UMR1037) for helpful discussions. English proofreading was performed by Greenland scientific proofreading.

\section{References}

1. Turner SD, Lamant L, Kenner L, Brugières L. Anaplastic large cell lymphoma in paediatric and young adult patients. $\mathrm{Br} \mathrm{J}$ Haematol. 2016;173(4):560-572.

2. Swerdlow SH, Campo E, Pileri SA, et al The 2016 revision of the World Health Organization classification of lymphoid neoplasms. Blood. 2016;127(20):2375-2390.

3. Morris SW, Kirstein MN, Valentine MB, et al. Fusion of a kinase gene, ALK, to a nucleolar protein gene, NPM, in non-Hodgkin's lymphoma. Science. 1994;263(5151):12811284.

4. Chiarle R, Voena C, Ambrogio C, Piva R, Inghirami G. The anaplastic lymphoma kinase in the pathogenesis of cancer. Nat Rev Cancer. 2008;8(1):11-23.

5. Werner MT, Zhao C, Zhang Q, Wasik MA. Nucleophosmin-anaplastic lymphoma kinase: the ultimate oncogene and therapeutic target. Blood. 2017;129(7):823-831.

6. Christensen JG, Zou HY, Arango ME, et al. Cytoreductive antitumor activity of PF2341066, a novel inhibitor of anaplastic lymphoma kinase and c-Met, in experimental models of anaplastic large-cell lymphoma. Mol Cancer Ther. 2007;6(12):33143322 .

7. Gambacorti Passerini C, Farina F, Stasia A, et al. Crizotinib in Advanced, Chemoresistant Anaplastic Lymphoma Kinase-Positive Lymphoma Patients. J Natl Cancer Inst. 2014;106(2):djt378.

8. Kruczynski A, Delsol G, Laurent C, Brousset P, Lamant L. Anaplastic lymphoma kinase as a therapeutic target. Expert Opin Ther Targets 2012; 16(11):1127-1138.

9. Sharma GG, Mota I, Mologni L, Patrucco E, Gambacorti-Passerini C, Chiarle R. Tumor
Resistance against ALK Targeted TherapyWhere It Comes From and Where It Goes. Cancers (Basel). 2018;10(3).

10. Frentzel J, Sorrentino D, Giuriato $S$ Targeting Autophagy in ALK-Associated Cancers. Cancers (Basel). 2017;9(12).

11. Feng Y, He D, Yao Z, Klionsky DJ. The machinery of macroautophagy. Cell Res. 2014;24(1):24-41

12. White E, DiPaola RS. The double-edged sword of autophagy modulation in cancer. Clin Cancer Res. 2009;15(17):5308-5316.

13. Galluzzi L, Pietrocola F, Bravo-San Pedro $\mathrm{JM}$, et al. Autophagy in malignant transformation and cancer progression. EMBO J. 2015;34(7):856-880.

14. Joffre C, Djavaheri-Mergny M, Pattingre S, Giuriato S. L'autophagie : le yin et le yang des cancers. Med Sci (Paris). 2017; 33(3):328-334.

15. Doherty J, Baehrecke EH. Life, death and autophagy. Nat Cell Biol. 2018; 20(10):1110-1117.

16. Lalaoui N, Lindqvist LM, Sandow JJ, Ekert PG. The molecular relationships between apoptosis, autophagy and necroptosis. Semin Cell Dev Biol. 2015;3963-3969.

17. Long JS, Ryan KM. New frontiers in promoting tumour cell death: targeting apoptosis, necroptosis and autophagy. Oncogene. 2012;31(49):5045-5060.

18. Yip KW, Reed JC. Bcl-2 family proteins and cancer. Oncogene. 2008;27(50):6398-6406.

19. Pattingre S, Tassa A, Qu X, et al. Bcl-2 antiapoptotic proteins inhibit Beclin 1-dependent autophagy. Cell. 2005;122(6):927-939.

20. Lindqvist LM, Heinlein M, Huang DCS, Vaux DL. Prosurvival Bcl-2 family members affect autophagy only indirectly, by inhibiting Bax and Bak. Proc Natl Acad Sci U S A. 2014;111(23):8512-8517.

21. Lindqvist LM, Vaux DL. BCL2 and related prosurvival proteins require BAK1 and BAX to affect autophagy. Autophagy. 2014; 10(8):1474-1475.

22. Pattingre $\mathrm{S}$, Levine $\mathrm{B} . \mathrm{Bcl}-2$ inhibition of autophagy: a new route to cancer? Cancer Res. 2006;66(6):2885-2888.

23. Souers AJ, Leverson JD, Boghaert ER, et al. ABT-199, a potent and selective BCL-2 inhibitor, achieves antitumor activity while sparing platelets. Nat Med. 2013;19(2):202208.

24. Mihalyova J, Jelinek T, Growkova K, Hrdinka M, Simicek M, Hajek R. Venetoclax: A new wave in hematooncology. Exp Hematol. 2018:61:10-25.

25. Tolcher AW, Rodrigueza WV, Rasco DW, et al. A phase 1 study of the BCL2-targeted deoxyribonucleic acid inhibitor (DNAi) PNT2258 in patients with advanced solid tumors. Cancer Chemother Pharmacol. 2014;73(2):363-371

26. Misso G, Di Martino MT, De Rosa G, et al. Mir-34: a new weapon against cancer? $\mathrm{Mol}$ Ther Nucleic Acids. 2014;3(9):e194.

27. Zarone MR, Misso G, Grimaldi A, et al. Evidence of novel miR-34a-based therapeutic approaches for multiple myeloma treatment. Sci Rep. 2017;7(1):17949.

28. Farooqi A, Tabassum S, Ahmad A. MicroRNA-34a: A Versatile Regulator of Myriads of Targets in Different Cancers. Int J Mol Sci. 2017;18(10).

29. Lamant L, Espinos E, Duplantier M, et al. Establishment of a novel anaplastic largecell lymphoma-cell line (COST) from a "small-cell variant" of ALCL. Leukemia. 2004;18(10):1693-1698.

30. Mitou G, Frentzel J, Desquesnes A, et al. Targeting autophagy enhances the antitumoral action of crizotinib in ALK-positive anaplastic large cell lymphoma. Oncotarget. 2015;6(30):30149-30164.

31. Villalva C, Bougrine F, Delsol G, et al. Bcl-2 expression in anaplastic large cell lym- 
phoma. Am J Pathol. 2001;158(5):18891890.

32. Rassidakis GZ, Sarris AH, Herling M, et al. Differential Expression of BCL-2 Family Proteins in ALK-Positive and ALK-Negative Anaplastic Large Cell Lymphoma of T/Null-Cell Lineage. Am J Pathol. 2001;159(2):527-535.

33. Fulda S, Kögel D. Cell death by autophagy: emerging molecular mechanisms and implications for cancer therapy. Oncogene. 2015;34(40):5105-5113.

34. Hosokawa N, Hara T, Kaizuka T, et al. Nutrient-dependent mTORC1 association with the ULK1-Atg13-FIP200 complex required for autophagy. Mol Biol Cell. 2009;20(7):1981-1991.

35. Saxton RA, Sabatini DM. mTOR Signaling in Growth, Metabolism, and Disease. Cell. 2017;168(6):960-976.

36. Mönch D, Bode-Erdmann S, Kalla J, et al. A subgroup of pleural mesothelioma expresses ALK protein and may be targetable by combined rapamycin and crizotinib therapy. Oncotarget. 2018;9(29):20781-20794.

37. Schläfli AM, Berezowska S, Adams O, Langer R, Tschan MP. Reliable LC3 and p62 autophagy marker detection in formalin fixed paraffin embedded human tissue by immunohistochemistry. Eur J Histochem. 2015;59(2):2481.

38. Holt S V., Wyspianska B, Randall KJ, James D, Foster JR, Wilkinson RW. The Development of an Immunohistochemical Method to Detect the Autophagy-Associated Protein LC3-II in Human Tumor Xenografts. Toxicol Pathol. 2011;39(3):516-523.

39. Rust R. High expression of Mcl-1 in ALK positive and negative anaplastic large cell lymphoma. J Clin Pathol. 2005;58(5):520524.

40. Desjobert C, Renalier MH, Bergalet J, et al. MiR-29a down-regulation in ALK-positive anaplastic large cell lymphomas contributes to apoptosis blockade through MCL-1 overexpression. Blood. 2011;117(24):66276637.

41. Yang F, Li Q, Gong Z, et al. MicroRNA-34a Targets Bcl-2 and sensitizes human hepatocellular carcinoma cells to sorafenib treatment. Technol Cancer Res Treat. 2013;13(1):77-86.

42. Li L, Yuan L, Luo J, Gao J, Guo J, Xie X. MiR-34a inhibits proliferation and migra- tion of breast cancer through down-regulation of Bcl-2 and SIRT1. Clin Exp Med. 2013;13(2):109-117.

43. Craig VJ, Tzankov A, Flori M, Schmid CA, Bader AG, Müller A. Systemic microRNA34a delivery induces apoptosis and abrogates growth of diffuse large B-cell lymphoma in vivo. Leukemia. 2012 26(11):2421-2424.

44. Scognamiglio I, Di Martino MT, Campan $\mathrm{V}$, et al. Transferrin-conjugated SNALPs encapsulating 2'-O-methylated miR-34a for the treatment of multiple myeloma. Biomed Res Int. 2014;2014:217365.

45. Beg MS, Brenner AJ, Sachdev J, et al. Phase I study of MRX34, a liposomal miR-34a mimic, administered twice weekly in patients with advanced solid tumors. Invest New Drugs. 2017;35(2):180-188.

46. Basit F, Cristofanon S, Fulda S. Obatoclax (GX15-070) triggers necroptosis by promoting the assembly of the necrosome on autophagosomal membranes. Cell Death Differ. 2013;20(9):1161-1173.

47. Sakamaki J, Ryan KM. Autophagy Determines the Path on the TRAIL to Death. Dev Cell. 2016;37(4):291-293. 